\title{
Advanced Integration of WiFi and Inertial Navigation Systems for Indoor Mobile Positioning
}

\author{
Frédéric Evennou and François Marx \\ Division R\&D, TECH/IDEA, France Telecom, 38243 Meylan, France \\ Received 23 June 2005; Revised 23 January 2006; Accepted 29 January 2006
}

\begin{abstract}
This paper presents an aided dead-reckoning navigation structure and signal processing algorithms for self localization of an autonomous mobile device by fusing pedestrian dead reckoning and WiFi signal strength measurements. WiFi and inertial navigation systems (INS) are used for positioning and attitude determination in a wide range of applications. Over the last few years, a number of low-cost inertial sensors have become available. Although they exhibit large errors, WiFi measurements can be used to correct the drift weakening the navigation based on this technology. On the other hand, INS sensors can interact with the WiFi positioning system as they provide high-accuracy real-time navigation. A structure based on a Kalman filter and a particle filter is proposed. It fuses the heterogeneous information coming from those two independent technologies. Finally, the benefits of the proposed architecture are evaluated and compared with the pure WiFi and INS positioning systems.
\end{abstract}

Copyright () 2006 Hindawi Publishing Corporation. All rights reserved.

\section{INTRODUCTION}

Mobile positioning becomes of increasing interest for the wireless telecom operators. Indeed, many applications require an accurate location information of the mobile (context-aware application, emergency situation, etc.). While many outdoor solutions exist, based on GPS/AGPS, in indoor environments, the received signals are too weak to provide an accurate location using those technologies. Currently, given that many buildings are equipped with WLAN access points (shopping malls, museums, hospitals, airports, etc.), it may become practical to use these access points to determine user location in these indoor environments. Moreover, new regulations will impose to VoWiFi (voice over WiFi) operators to integrate a positioning solution in their terminals to comply with the E911 policy [1]. The location technique is based on the measurement of the received signal strength (RSS) and the well-known fingerprinting method $[2,3]$. The accuracy depends on the number of positions registered in the database. Besides, signal fluctuations over time introduce errors and discontinuities in the user's trajectory.

To minimize the fluctuations of the RSS, some filtering is needed. A simple temporal averaging filter does not give satisfying results. Kalman filtering $[4,5]$ is commonly used in automatic control to track the trajectory of a target. However, more information can be used to improve the accuracy. In the following sections, we choose to use a map of the environment. It is used in order to find the most probable trajectory of the mobile and avoid wall crossings. Including such information requires new filters as the Kalman filter is not adapted for this. Particle filters [6-8], based on MonteCarlo simulations, are emerging to solve the problems of position estimation.

Inertial navigation systems (INS) are one of the most widely used dead-reckoning systems. They can provide continuous position, velocity, and also orientation estimates, which are accurate for a short term, but are subject to drift due to noise of the sensor $[9,10]$. Filtering techniques will limit the effect of the measurement noise and therefore reduce this drift. The Kalman filter is already used in many GPS/INS applications, to reduce the effect of this measurement noise. Merging positioning information from two so different technologies must lead to very interesting results. Moreover, the strength of the INS system should annihilate the weaknesses of WiFi and vice versa. Those heterogenous but complementary technologies should lead to an enhanced system in terms of positioning performance as well as availability of the positioning service over a larger area. Indeed, when the WiFi positioning is unavailable because of network uncovered area, the dead-reckoning system can go on and provide a position estimate which is degraded over the time but can be reliable over a certain period.

This paper presents in its second section the basic techniques leading to a first estimate of the position of a WiFi device thanks to the associated network. The third section introduces a convenient way based on the use of the particle 
filter to reduce the effect of the WiFi measurement noise and to integrate more information such as the map of a building. Section 4 presents our system based on dead-reckoning navigation, and will use information from a dual axis accelerometer, a gyroscope, and a pressure sensor. The next section demonstrates the capability of the particle filter to integrate information of those two different technologies and combine them efficiently to lead to a more performing system. Finally, Section 6 gives some information about the performance of all those different systems, when used separately, and cooperatively.

\section{BASIC INDOOR MOBILE POSITIONING WITH WIFI}

Many outdoor systems are based on time measurements, that is, the mobile equipment and the network are synchronized. Thus, the mobile can calculate its distance from the access point (AP).

However getting this kind of information with off-theshelf WiFi equipments is almost impossible. The only available information is the signal strength received from each AP. Indeed, the received signal strength is measured and is one of the outputs of the card. Such information is available because the APs send beacons periodically. Mobile devices use those beacons to handle the roaming inside the network. Given this consideration, it is possible to get a list of the received power coming from all the APs covering the area where the mobile is moving.

\subsection{Signal strength and propagation model}

The reception of a tuple of signal strengths does not lead directly to the position of the device. A conversion of this tuple of received signal strengths into a position is required. The Motley-Keenan propagation model is a convenient propagation model often used for its simplicity. This model is presented in [11]; its simplest form is given by

$$
P_{\text {received }}(d)=P_{\text {received }}\left(d_{0}\right)-10 \cdot \alpha \cdot \log \left(\frac{d}{d_{0}}\right)
$$

where $P_{\text {received }}(d)$ is the signal strength received by the mobile at distance $d, P_{\text {received }}\left(d_{0}\right)$ the signal strength received at the known distance $d_{0}$ from the AP, and $\alpha$ a coefficient modeling the radio wave propagation in the environment. For example, in free path loss environment, we have $\alpha=2$. In indoor environments, this factor will be closer to 3 [12].

This model is rather simple and needs only two parameters, that is, $P_{\text {received }}\left(d_{0}\right)$ and $\alpha$. Ranging experiments were carried out using this propagation model, but a very poor accuracy was obtained, probably due to the too simple form of this model, in comparison to the complex radio environment.

Refinements of this model exist. They introduce some wall attenuation factors, but some extra information is needed [3] to describe more closely the environment. The walls' materials must be characterized, and their properties must be introduced in the model, leading to the following approximation [3]:

$$
P_{\text {received }}(d)=P_{\text {received }}\left(d_{0}\right)-10 \cdot \alpha \cdot \log \left(\frac{d}{d_{0}}\right)+\sum_{i=0}^{N_{w}} n_{i} \cdot \omega_{i} \text {, }
$$

where $N_{w}-1$ is the number of walls of different nature, $n_{i}$ is the number of walls having an attenuation of $\omega_{i} \mathrm{~dB}$. Such a propagation model leads to a better estimate of the range separating the mobile from each AP, but requires more efforts to calibrate. Combining those estimated ranges with a multilateration algorithm, it is possible to find the position of the mobile.

Further investigations showed that introducing the estimated ranges, obtained with the propagation models described above, in a multilateration algorithm leads to a poor positioning due to the large estimation errors. Those errors appear because the propagation models are too simple in comparison to the complex indoor RF propagation.

\subsection{WiFi cell ID, signal strength and fingerprinting}

The simplest approach for locating a mobile device in a WLAN environment is to approximate its position by the position of the access point received at that position with the strongest signal strength. The major benefit of such a system is its simplicity, but its main drawback is its large estimation error. The accuracy is proportional to the density of access points, which is in the range of 25 to 50 meters for indoor environments [13]. Reference [2] introduced a different approach for locating the device in indoor environments by using the radio signal strength fingerprinting.

Fingerprinting positioning is a quite different technique. It consists in having some signal power footprints or signatures that define a position in the environment. This signature is made of the received signal powers from different access points that cover the environment. A first step, called training or profiling, is necessary to build this mapping between collected received signal strength and certain positions in the building. This leads to a database that is used during the positioning phase. Building the footprint database can be done in two ways. A first method is to do on-site measurements for some reference positions in the building with a user terminal. An alternative approach is based on collecting limited on-site measurements and introducing them in a tunable propagation model that would use them to fit some of its parameters. Then, this propagation model gives an extensive coverage map for each AP. However, the poor results obtained earlier with the use of the propagation model did not invite us to focus on such a model. Neural networks are another learning method for improving propagation models over time [14]. It was decided to carry on with the use of the data collected to build the database. Ray tracing tools represent another solution to build such a database, but they are very complex tools. Moreover, a good knowledge of the radio environment (knowledge of the presence and position of all the APs) is needed to cope with the interfering issue. 
However, such information is not always available due to the fast growing emergence of this technology in indoor environments.

Once this prerequisite step is accomplished, it is necessary to do the reversing operation, which will deliver the position associated to an instantaneous collected tuple of received signal strengths. Different techniques can fit these requirements.

\subsection{1. $\quad k$-closest neighbors fingerprinting}

This algorithm goes through the database and picks the $k$ referenced positions that match best the observed received signal strength tuple. The criterion that is commonly retained is the Euclidian distance (in signal space) metric. If $Z=\left[\mathrm{RSS}_{1}, \ldots, \mathrm{RSS}_{M}\right]$ is the observed RSS vector composed of $M$ received access points at the unknown position $X=(x, y)$ and $Z_{i}$ the footprint recorded in the database for the position $X_{i}=\left(x_{i}, y_{i}\right)$, then this Euclidian distance is

$$
d\left(Z, Z_{i}\right)=\frac{1}{M} \cdot \sqrt{\sum_{j=1}^{M}\left(\operatorname{RSS}_{j}(x, y)-\mathrm{RSS}_{j}\left(x_{i}, y_{i}\right)\right)^{2}},
$$

where $\mathrm{RSS}_{j}\left(x_{i}, y_{i}\right)$ is the mean value recorded in the database for the access point whose MAC address is noted " $j$ " at the position $\left(x_{i}, y_{i}\right)$.

The set $N_{k}$ of the database positions having the smallest errors is built with an iterative process as follows:

$$
N_{k}=\left\{\underset{X_{i} \in \mathfrak{L}}{\operatorname{argmin}}\left[d\left(Z, Z_{i}\right)\right] \backslash X_{i} \notin N_{k-1}\right\} \text {, }
$$

where $\mathfrak{L}$ is the set of positions recorded in the database. This set contains $k$ positions. Finally, the position of the mobile is considered to be the barycenter of those $k$ selected positions:

$$
X=\frac{\sum_{j=1}^{k}\left(1 / d\left(Z, Z_{i}\right)\right) \cdot X_{j}}{\sum_{j=1}^{k}\left(1 / d\left(Z, Z_{i}\right)\right)} \quad \text { with } X_{j} \in N_{k} .
$$

The main advantage of this method is its simplicity to set it up. However the accuracy highly depends on the granularity of the reference database [15]. A better accuracy can be achieved with finer grids, but a finer grid means a larger database that is more time costly.

\subsubsection{Probabilistic estimation}

The main drawback of the nearest neighbor method is its lack of accuracy when the size of the database is limited. A probabilistic approach has been proposed in [16, 17]. This approach is based on an empirical model that describes the distribution of received signal strength at various locations. The use of probabilistic models provides a natural way to handle uncertainty and errors in signal power measurements. Thus, after the calibration phase, for any given location $X$, a probability distribution $\operatorname{Pr}[Z \mid X]$ assigns a probability for each measured signal vector $Z$. Applying the Bayes rule leads to the following posterior distribution of the location [16]:

$$
\begin{aligned}
\operatorname{Pr}[X \mid Z] & =\frac{\operatorname{Pr}[Z \mid X] \cdot \operatorname{Pr}[X]}{\operatorname{Pr}[Z]} \\
& =\frac{\operatorname{Pr}[Z \mid X] \cdot \operatorname{Pr}[X]}{\sum_{X_{i} \in \mathfrak{L}} \operatorname{Pr}\left[Z \mid X_{i}\right] \cdot \operatorname{Pr}\left[X_{i}\right]},
\end{aligned}
$$

where $\operatorname{Pr}[X]$ is the prior probability of being at location $l$ before knowing the value of the observation variable, and the summation goes over the set of possible location values, denoted by $\mathfrak{L}$.

The prior distribution $\operatorname{Pr}[X]$ gives a simple way to incorporate background information, such as personal user profiles, and to implement tracking. In case neither user profiles nor a history of measured signal properties allowing tracking are available, one can simply use a uniform prior which introduces no bias towards any particular location. As the denominator $\operatorname{Pr}[Z]$ does not depend on the location variable $l$, it can be treated as a normalizing constant whenever only relative probabilities or probability ratios are required.

The posterior distribution $\operatorname{Pr}[X \mid Z]$ can be used to choose an optimal estimator of the location based on whatever loss function is considered to express the desired behavior. For instance, the squared error penalizes large errors more than small ones, which is often useful. If the squared error is used, the estimator minimizing the expected loss is the expected value of the location variable:

$$
E[X \mid Z]=\sum_{X_{i} \in \mathfrak{L}} l \cdot \operatorname{Pr}[X \mid Z]
$$

assuming that the expectation of the location variable is well defined, that is, the location variable is numerical. Location estimates, such as the expectation, are much more useful if they are complemented with some indication about their precision.

However, in both techniques, the signal strength fluctuations (Figure 1) introduce many unexpected jumps in the final trajectory. Removing those jumps can be done by using a filter. Kalman filter and particle filter are often used in parameter estimating problems and tracking. This last filter will be introduced in the next section, and the benefits using such a filter will be presented.

\section{IMPROVING WIFI POSITIONING WITH A PARTICLE FILTER}

Nowadays, the maps of all the public or company buildings are available in digital format (dxf, jpeg, etc.). The key idea is to combine the motion model of a person and the map information in a filter in order to obtain a more realistic trajectory and a smaller error for a trip around the building. In the following, it will be considered that the map, which is available, is a bitmap. So no information is available except the pixels in black and white which model the structure of the building. The particle filter, based on a set of random weighted samples (i.e., the particles), represents the density function of the mobile position. Each particle explores the environment according to the motion model and map information. 


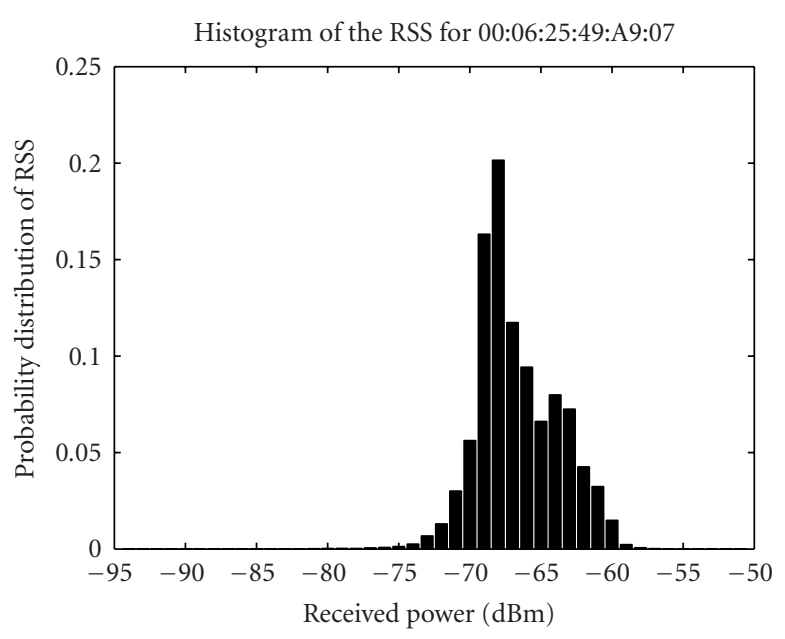

(a)

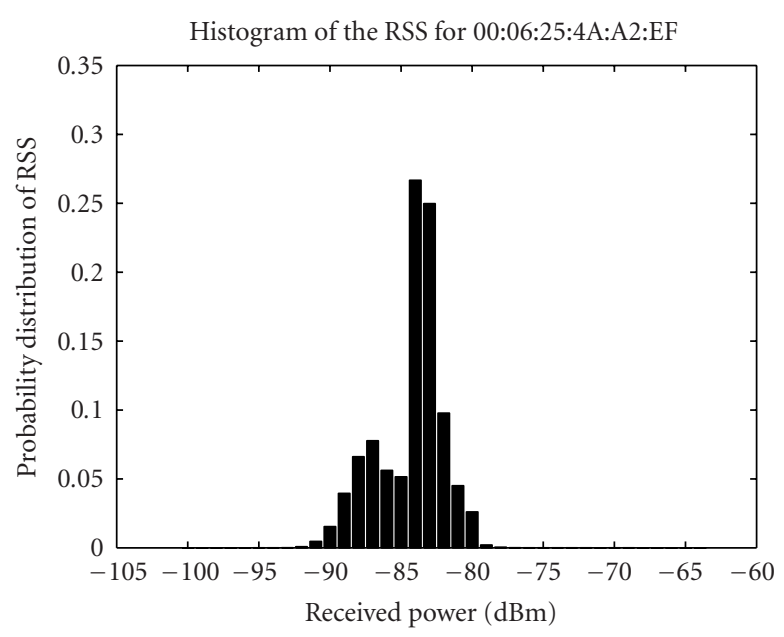

(b)

FIGURE 1: Received signal strength variations over time for two different access points and for the same position.

Their weights are updated each time a new measurement is received. It is possible to forbid some moves like crossing the walls by forcing the weight at 0 for the particles having such a behavior.

The particle filter tries to estimate the probability distribution $\operatorname{Pr}\left[x_{k} \mid Z_{0: k}\right]$, where $x_{k}$ is the state vector of the device at the time step $k$, and $Z_{0: k}$ is the set of collected measurements until the $(k+1)$ th measurement. When the number of particles (position $x_{k}^{i}$, weight $w_{k}^{i}$ ) is high, the discrete probability density function of presence can be assimilated to

$$
\operatorname{Pr}\left[x_{k} \mid Z_{0: k}\right]=\sum_{i=1}^{N_{s}} w_{k}^{i} \delta\left(x_{k}-x_{k}^{i}\right) .
$$

This filter comprises two steps:

(i) prediction;

(ii) correction.

\subsection{Prediction}

During this step, the particles propagate across the building given an evolution law that assigns a new position for each particle with an acceleration governed by a random process:

$$
\begin{aligned}
{\left[\begin{array}{l}
x_{k+1} \\
y_{k+1} \\
v_{x_{k+1}} \\
v_{y_{k+1}}
\end{array}\right]=} & {\left[\begin{array}{cccc}
1 & 0 & T_{s} & 0 \\
0 & 1 & 0 & T_{s} \\
0 & 0 & 1 & 0 \\
0 & 0 & 0 & 1
\end{array}\right]\left[\begin{array}{l}
x_{k} \\
y_{k} \\
v_{x_{k}} \\
v_{y_{k}}
\end{array}\right] } \\
& +\left[\begin{array}{cccc}
\frac{T_{s}^{2}}{2} & 0 & 0 & 0 \\
0 & \frac{T_{s}^{2}}{2} & 0 & 0 \\
0 & 0 & T_{s} & 0 \\
0 & 0 & 0 & T_{s}
\end{array}\right]\left[\begin{array}{l}
\eta_{x_{k}} \\
\eta_{y_{k}} \\
\eta_{x_{k}} \\
\eta_{y_{k}}
\end{array}\right],
\end{aligned}
$$

where $\left[x_{k}, y_{k}, v_{x_{k}}, v_{y_{k}}\right]^{T}$ denotes the state vector associated to each particle (position and speed), $T_{s}$ the elapsed time between the $(k-1)$ th and the $k$ th WiFi measurements. $\left[\eta_{x_{k}}, \eta_{y_{k}}, \eta_{x_{k}}, \eta_{y_{k}}\right]^{T}$ is a random process that simulates the acceleration of the $k$ th particle. This last equation is often called the prior equation. It has the form of the movement law (Newton's laws) given by $x_{k}=x_{k-1}+v \cdot T_{s}+a \cdot T_{s}^{2} / 2$, where $a$ is the acceleration of the mobile and $v$ its velocity. Here the particles are given a random exploration move thanks to the acceleration random process. It tries to predict a new position for all the particles. The used process is a zero mean Gaussian noise whose variance must be realistic of a pedestrian movement.

When the new position of a particle is known, it is possible to include the map information, in order to remove the particles having an impossible move, like crossing a wall. An algorithm, using the previous known position of the particle, its new one, plus the map of the building, checks all the pixels between those positions to see if a wall has been crossed. This processing is time consuming as it must be done for each particle at each time step. When this checking is finished, it is possible to assign a weight $\operatorname{Pr}\left[x_{k} \mid x_{k-1}\right]$ as follows:

$$
\operatorname{Pr}\left[x_{k} \mid x_{k-1}\right]= \begin{cases}P_{m} & \text { if a particle crossed a wall, } \\ 1-P_{m} & \text { if a particle did not cross a wall. }\end{cases}
$$

Since crossing a wall is impossible for a normal user, it has been decided to take $P_{m}=0$. Then, the particles disappear when they cross a wall. A common problem with the particle filter is the degeneracy phenomenon: after a few iterations, many particles will have a negligible weight. A resampling step will occur when the degeneracy is too severe (see Section 3.4).

\subsection{Correction}

When a measurement (tuple of RSS) is available, it must be taken into account to correct the weight of the particles in order to approximate $\operatorname{Pr}\left[x_{k} \mid Z_{0: k}\right]$. As the measurement is made of signal strengths and given that particles are characterized by their position, the RSS tuple must be transformed 
into a position. The mapping between the position and the signal strengths is performed thanks to the empirical fingerprinting database. In fact, the algorithm used in Section 2.2 to find the position of the mobile, given the RSS coverage in the building, is used. Then it is possible to estimate $\operatorname{Pr}\left[Z_{k} \mid x_{k}\right]$. In the case of an indoor movement, the closest neighbor algorithm returns a position denoted $X_{z_{k}}$ (see Section 2.2.1), which matches the current WiFi measurement. This last position, equivalent to the measurement, is introduced in the weight of the particles as follows:

$$
\operatorname{Pr}\left[Z_{k} \mid x_{k}^{i}\right]=\frac{1}{\sqrt{2 \pi} \sigma} \exp \left[-\frac{\left\|X_{z_{k}}-X_{x_{k}^{i}}\right\|^{2}}{2 \cdot \sigma^{2}}\right]
$$

with $X_{z_{k}}$ being the position returned by the database, $X_{x_{k}^{i}}$ the position of the $i$ th particle at time step $k$, and $\sigma$ the measurement confidence. The smaller $\sigma$ will be, the more confident the user is in the measurement. That would mean that there are very little variations in the measurements for the same position. Here, $\sigma$ is chosen depending on the variations of the RSS. It can be noticed that with this Gaussian law, the closer the particle is to the position returned by the database, the higher its $\operatorname{Pr}\left[Z_{k} \mid x_{k}\right]$ will be. Now, having defined all the necessary probabilities to update the weight of a particle, we just need to combine them to find the new posterior distribution.

\subsection{Particle update}

The weight update equation is given in $[6,7]$

$$
w_{k}^{i}=w_{k-1}^{i} \cdot \operatorname{Pr}\left[x_{k} \mid x_{k-1}\right] \cdot \operatorname{Pr}\left[z_{k} \mid x_{k}\right] .
$$

To obtain the posterior density function, it is necessary to normalize those weights. After a few iterations, when too many particles crossed a wall, just a few particles will be kept alive (particles with a nonzero weight). To avoid having just one remaining particle, a resampling step is triggered.

\subsection{Resampling}

The resampling is a critical point for the filter. The basic idea behind the resampling step is to move the particles that have a too low weight, in the area of the map where the highest weights are. This leads to a loss of diversity because many samples will be repeated. The criterion to trigger a resampling is given by

$$
\frac{1}{\sum_{i=0}^{N_{s}}\left(w_{k}^{i}\right)^{2}} \leq \text { Threshold. }
$$

Various resampling algorithms were proposed. We did not choose the simple SIS (sequential importance sampling) particle filter [6], but the resampling approach presented in [18]: the regularized particle filter (RPF). The RPF adds a regularization step. This approach is more convenient because it locally introduces a new diversity after the resampling. This may be useful in extreme situations when all the particles are trapped in a room; whereas the device is still moving along a corridor. This method of resampling adds a small noise to the particle position and avoids this phenomenon.

The main stages of the particle filter used in indoor environments have been presented. To run it, a large number of particles must be used. This makes the filter very heavy to process at each time step as every particle must be checked for a wall crossing. Due to the large number of particles, the algorithm is too complex to be implemented on handheld devices. A way to cut down this number of particles must be found. Using a new representation of the building is one of the solutions. The Voronoi diagram of the building has been used in $[19,20]$ to reduce the computation complexity of the particle filter.

\section{POSITIONING WITH INERTIAL NAVIGATION SENSORS}

INSs are self-contained, nonradiating, nonjammable, deadreckoning navigation systems which provide dynamic information through direct measurements. Fundamentally, gyroscopes provide angular rate, and accelerometers provide velocity rate information. Although the information rates are reliable over long periods of time, they must be integrated to provide orientation, linear position, and velocity information. Thus, even very small errors in the information rates can cause an unbounded growth in the error of integrated measurements. One way of overcoming this problem is to use inertial sensors in conjunction with other absolute sensing mechanisms to periodically reset them.

In this experiment, the available sensors are: a gyroscope that delivers some information about the angular speed of the mobile; a biaxial accelerometer to count the number of steps, and to detect if it is moving or not; and the last sensor is an atmospheric pressure sensor, used in detecting when the mobile is going from one floor to the other. Other sensors, like magnetometers, could be added. In order to collect some relevant measurements translating the real moves of the pedestrian, the sensing box needs to be attached to a part of the body that is only affected by the moves of the user. The belt (or the hips) is an interesting part of the body for collecting information about the behavior of the user.

Interests in such a positioning technology increase because mobile phones start integrating such systems [21]. Users often have their mobile phone at the belt, so it would be possible to use those sensors in order to get an estimate of their position.

Here the accelerometer has been used to count the number of steps the user did during his trajectory. This is possible because when the user is walking the signal fluctuates periodically as long as he keeps moving at the same speed. Using a thresholding system, it becomes possible to accurately estimate the number of steps the user did. Getting an estimate of the distance is tougher as it requires a calibration step, and the hypothesis that all the user's steps stride are always the same. However, over a certain distance, such an assumption seems realistic.

To keep track of the rotation around the $z$-axis, the angular velocity $\omega$ sensed by the gyroscope must be integrated. 
Defining $\theta_{z}$ as the current $z$-axis relative to the original orientation, we have

$$
\theta_{z}=\int_{0}^{T} \omega_{z} d t
$$

With this information, it becomes possible to predict the position of the mobile at each time step, given that the initial position of the mobile is known when the inertial navigation sensors are powered. This position is given by

$$
\left[\begin{array}{l}
x_{k} \\
y_{k}
\end{array}\right]=\left[\begin{array}{l}
x_{k-1} \\
y_{k-1}
\end{array}\right]+v \cdot \Delta T \cdot\left[\begin{array}{c}
\cos \left(\theta_{k}\right) \\
\sin \left(\theta_{k}\right)
\end{array}\right],
$$

where $v$ is the speed of the mobile resulting from the product of the step stride and the step frequency, $\Delta T$ the elapsed time between two angular speed measurements. The step frequency is obtained thanks to the data coming from the accelerometer sensor. Future generation of the system should be able to estimate this parameter on the fly. $\theta_{k}$ is the rotation along the $z$-axis that occurred during the move of the pedestrian. This last parameter is obtained from the gyroscope:

$$
\theta_{k}=\sum_{t=0}^{k}\left(\dot{\theta}_{k}-\dot{\theta}_{k-1}\right) \Delta T
$$

However, a more realistic model must take into account the measurement noise. This noise represents the weakness of dead-reckoning positioning system. The quality of this system is related to the quality of the sensors that are integrated. Indeed, the power of this noise is quite important, as it generates a deviation on the trajectory. This drift needs to be corrected in order to avoid such errors.

Here a 2D problem has been considered, but it is possible to get the third coordinate of the mobile. The atmospheric pressure sensor incorporated in the sensor box can be used to measure the pressure variations. Pressure variations are relevant over a short period. It becomes inconsistent over a long period as the pressure can change naturally due to the weather. Thus measuring those variations will lead to know if the mobile is climbing or going down, and it is possible to know the elevation of the mobile with the equations described in [22-24].

Inertial navigation is a dead-reckoning technique, which suffers from one serious limitation: drift rate errors constantly accumulating over time. Since its drift errors relentlessly accumulate, an inertial navigation system that operates for an appreciable length of time must be updated periodically with fresh positioning information. This can be accomplished by using an external navigation reference, such as WiFi positioning.

\section{COOPERATION BETWEEN INS NAVIGATION AND WIFI POSITIONING SYSTEMS}

Combination of GPS and inertial navigation sensors is common in automotive applications in order to extend the coverage of GPS, as dead reckoning keeps delivering the position of the mobile during GPS unavailability periods. For the WiFi positioning system presented above, the interest is to get a better knowledge of the behavior of the mobile in order to reduce the effect of the WiFi measurement noise, and to guide the particles in a smarter way. Combining information coming from those two heterogeneous technologies must lead to performance improvements for the WiFi positioning system presented in Section 2, as the behavior of the particles could be refined with the INS sensors measurements. To optimally combine the redundant INS information, a Kalman filtering scheme is used whereby WiFi measurements regularly update the inertial state vector. A system combining the power of the WiFi positioning system using a particle filter, with the filtered INS information coming from a Kalman filter used to track the INS information, can be suitable to improve the whole positioning of the mobile.

The form of the particle filter is convenient to introduce the information coming from the INS sensors. This information can guide the particles as they are directly related to the behavior of the user.

On the other hand, the use of a Kalman filter for the INS sensors information, particularly for the information coming from the gyroscope, makes it possible to reduce the effect of the noise affecting this sensor, as the trajectory of the barycentre of the particles (including the map information) can be injected in the Kalman filter to correct this drift. Figure 2 presents the architecture that has been implemented to realize an indoor WiFi/INS positioning demonstrator.

Here the smoothing filter is the particle filter and the data filtering box corresponds to the processing that data coming from the INS sensors undergo. Integrating the information coming from the inertial navigation sensor inside the particle filter seems quite easy as it just requires to change the prediction (9) as follows:

$$
\begin{aligned}
{\left[\begin{array}{l}
x_{k+1} \\
y_{k+1}
\end{array}\right]=} & {\left[\begin{array}{ccc}
1 & 0 & T_{s} \cdot \cos \left(\theta_{k}\right) \\
0 & 1 & T_{s} \cdot \sin \left(\theta_{k}\right)
\end{array}\right]\left[\begin{array}{l}
x_{k} \\
y_{k} \\
v_{k}
\end{array}\right] } \\
& +\left[\begin{array}{cc}
\frac{T_{s}^{2}}{2} & 0 \\
0 & \frac{T_{s}^{2}}{2}
\end{array}\right]\left[\begin{array}{l}
\eta_{x} \\
\eta_{y}
\end{array}\right]
\end{aligned}
$$

with $v_{k}$ the amplitude of the speed estimated thanks to the data coming from the accelerometer sensor. $\theta_{k}$ is the angle returned by the inertial navigation processing unit. This angle is obtained thanks to the Kalman filter that uses the data coming from the gyroscope and the angle of the trajectory delivered by the WiFi positioning system. The following set of equations presents the Kalman filter that is used in this application to track the rotation of the mobile:

$$
\begin{gathered}
\theta_{k}^{-}=\theta_{k-1}-\dot{\theta}_{k} \cdot \Delta T, \\
P_{k}^{-}=\mathbf{Q}+P_{k-1}, \\
K_{k}=P_{k}^{-} \cdot\left[P_{k}^{-}+\mathbf{R}\right]^{-1}, \\
\theta_{k}=\theta_{k}^{-}+K_{k}\left[\theta_{\text {trajectory }}-\theta_{k}^{-}\right], \\
P_{k}=\left(1-K_{k}\right) \cdot P_{k}^{-}
\end{gathered}
$$

with $\dot{\theta}_{k}$ being the angular speed returned by the gyroscope, $\theta_{k-1}$ the previous predicted angle, $\Delta T$ the time between two 


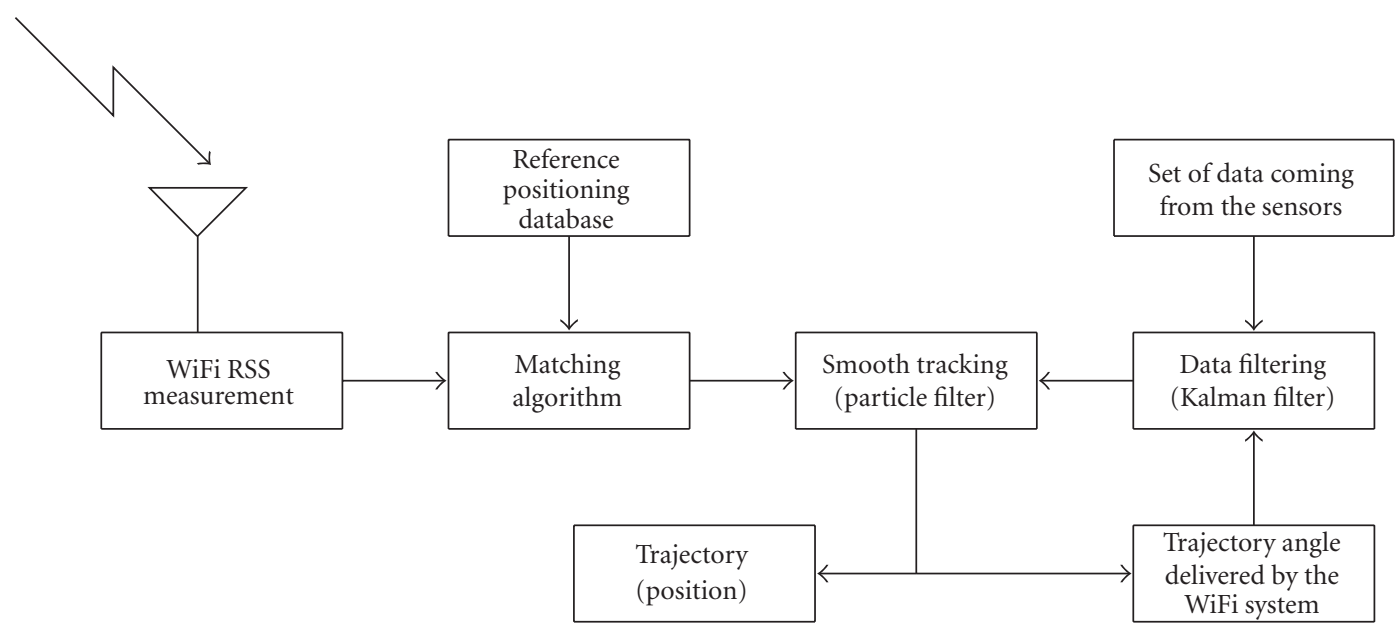

FIGURE 2: Block diagram of the INS/WiFi mutually correcting architecture.

measurements from the inertial sensors, as well as $\mathbf{Q}$ and $\mathbf{R}$ the covariance matrixes of noises affecting the process and measurement equations, respectively, describing the Kalman filter. $K_{k}$ represents the Kalman gain. $P_{k}^{-}$and $P_{k}$ denote the error covariance matrixes, and $\theta_{\text {trajectory }}$ is the angle of the trajectory returned by the particle filter, related to the $\mathrm{WiFi}$ measurements.

This structure enables sensors to correct one another in a smart manner. However, if a sensor fails (WiFi due to a degraded fingerprinting database), then the whole system will fail to provide a good estimate of the position of the device as the system is mainly based on the WiFi positioning. On the other hand, a failure for the INS system will be less stringent. Data from INS sensors are just used to indicate the behavior that the particle must follow. This will lead to make the particles moving in the wrong direction, and then the filter will trigger a resampling step to concentrate the particles in the most interesting areas where the mobile is standing. Such a resampling step will be triggered more often than normal. Thus, failure of the INS system will lead to a degradation of the positioning, but will not blind the system. If the WiFi system fails to give a correct position then the INS system will not be able to correct the whole system.

The next section presents the results that are obtained by using all these techniques.

\section{PERFORMANCE EVALUATION BASED ON EXPERIMENTAL RESULTS}

Experimentations were conducted to get a better idea of the performances that can be awaited from such positioning techniques. Experimentations were carried out in a $40^{\star} 40 \mathrm{~m}$ indoor office building. An access point was standing in each corner of this building. The mobile terminal was a laptop on which all these algorithms were running. A box containing all the INS sensors was sending the data frames built by a microcontroller to the PC via a RS232 interface. This box was attached to the belt of the pedestrian who needed his position while moving through the building. The sensors used in our box to collect some user behavioral information are: the ADXRS150 to get the angular speed (sensitivity: $\pm 150^{\circ} / \mathrm{s}$, rate noise density: $0.05^{\circ} / \mathrm{s} / \sqrt{\mathrm{Hz}}$ ), the dual axis ADXL202 to measure the vertical acceleration (detection if the mobile is moving or not) (range: $\pm 2 \mathrm{~g}$, noise density: $500 \mu \mathrm{g} / \sqrt{\mathrm{Hz}}$ ), and the MPX4115A barometer sensor measuring the atmospheric pressure (range: $15-115 \mathrm{kPa}$, sensitivity: $45.9 \mathrm{mV} / \mathrm{kPa}$ ).

The signal strength database is built with one measurement in each room, and a measurement every two meters in the corridor. The single floor problem is considered. A walk around the building is taken for the test. Some real measurements are collected along this path and then reused to estimate the performances of each technique. WiFi measurements were collected every $T_{s}=300 \mathrm{~ms}$, and a new INS measurement is available every $\Delta T=40 \mathrm{~ms}$. In all the tests, the mobile is moving at a regular walking speed of $1 \mathrm{~m} / \mathrm{s}$. Higher speed can be handled by the filter because the speed of the particles adapts itself over the time given the WiFi measurements. To get an overview of the highest acceptable speed of the device localized by the system, we must take into account the range between the elements (center of the rooms, corridor) of the environment. Here it is about $3 \mathrm{~m}$. As we collect WiFi measurements every $300 \mathrm{~ms}$, we can consider that a limit speed would be $3 / 0.3=10 \mathrm{~m} / \mathrm{s}$.

A first experiment (Figure 3) was carried out to compare the performances of the two fingerprinting algorithm presented in Section 2.2.

The vectors on the map represent the instantaneous error for each position of the trajectory corresponding to a $\mathrm{WiFi}$ measurement. The length of the vectors represents the instantaneous RMS error (comparison with the rebuilt "real" trajectory obtained assuming that the mobile moves at a constant speed on straight parts of the trajectory). The direction of the arrow indicates if the estimation was delayed or advanced in comparison to the real position of the mobile. 


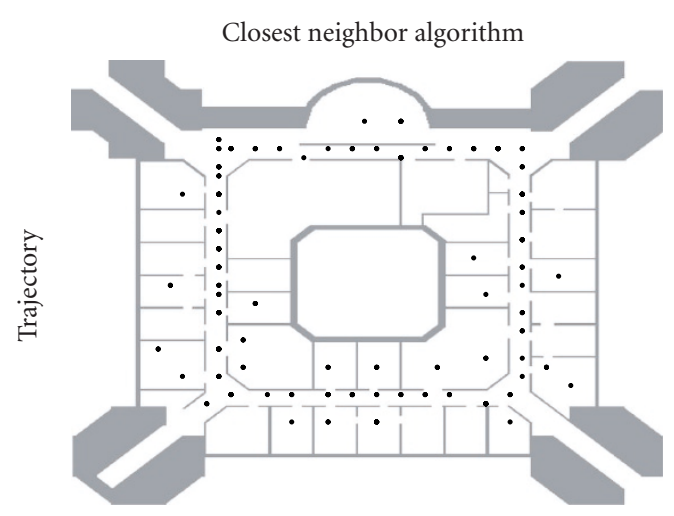

(a)

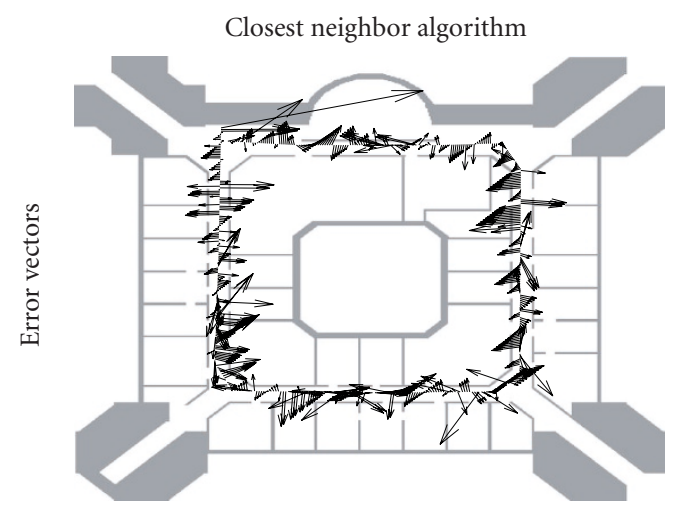

(c)

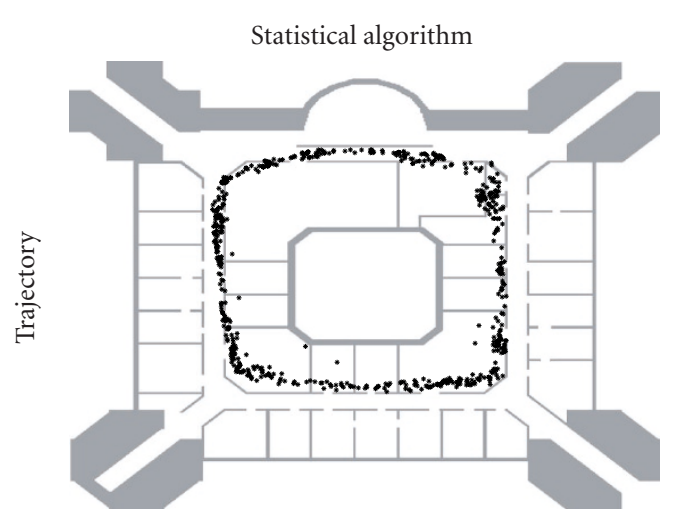

(b)

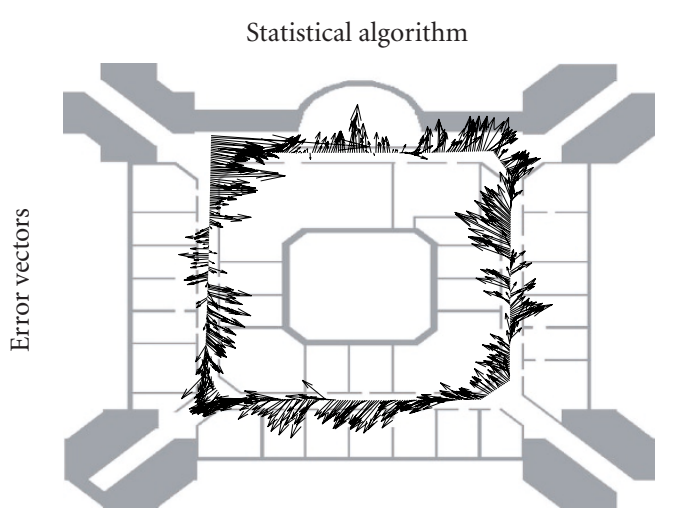

(d)

FIgURE 3: Trajectories comparison between the closest neighbor algorithm (Section 2.2.1) and the probabilistic position estimation (Section 2.2.2).

It appears that the performance with the probabilistic approach leads to slightly more accurate results over the trajectory. This is normal as the information, the probability distribution function of the RSS that is used, is richer than the simple mean RSS value. A good point for this method is that with a sparse database, not following a regular mesh, it is possible to get a 3-meter accuracy positioning. Other tests using more access points were carried out. They showed that the performance could be improved with more access points in the environment, and the redundancy introduced by those access points seems to be a good way to fight the error caused by the radio interferences which could create some identical footprints if not enough access points are considered.

However, in both techniques, we can notice that some jumps from one measurement to the other are present on the trajectory. Applying the particle filter, with 10000 particles, and combining the map information and the WiFi measurements (Figure 4), reduce the jumps introduced by the noisy measurements on the one hand, and on the other hand, it is possible to guess the trajectory of the user when walking through the building. Indeed, the moves of the user remain between the walls, and appear to be more realistic. But a little time delay can be observed on the final trajectory especially when decisions need to be done when the filter has several choices (choice between two rooms whose doors are in front of one another), or when the user abruptly changes his trajectory (entering a room); the filter keeps going ahead without changing quick enough its parameters. Then the filter's inertia can be observed and could be a little bit disturbing for the final user. Using the INS system on its own in indoor environments was tested. Figure 5 presents a trajectory through the corridor that was obtained by just taking into account the angular velocity and an estimate of the mean distance of the user's step. It can be noticed that the trajectory is quite steady, without any jumps. The true direction changes are clearly detected, and seem to be well estimated. But during straight moves, the noise affecting the sensors seems to be damageable. Indeed, an important drift is present and needs to be corrected prior to final implementation. However, the sensors seem to be quite accurate, especially when estimating the angular speed of the mobile. It is possible to estimate the angle the user turned within some few degrees. Thus combining this system with the particle filter should improve the estimation of the user's position.

The same trajectories were followed, but this time both navigation systems were enabled (Figure 6). The left column 


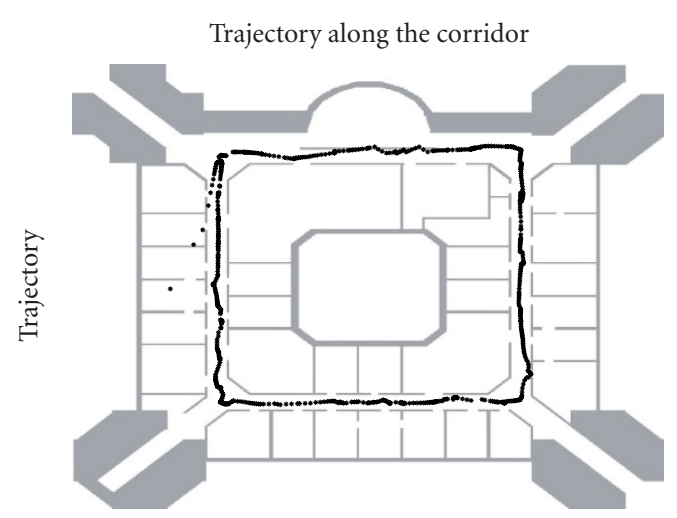

(a)

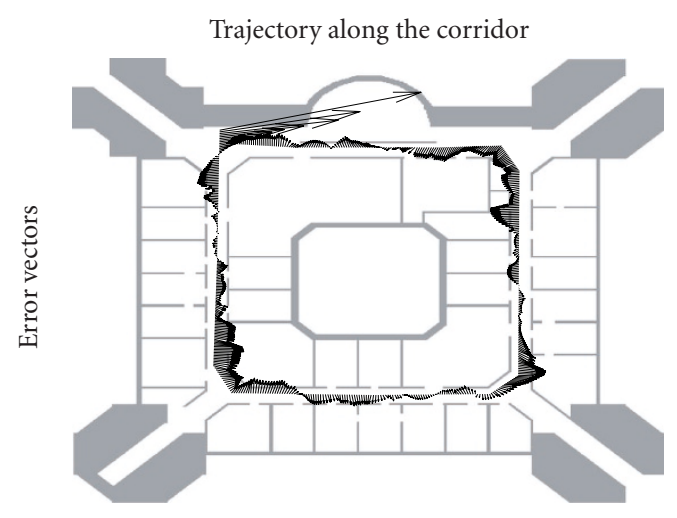

(c)

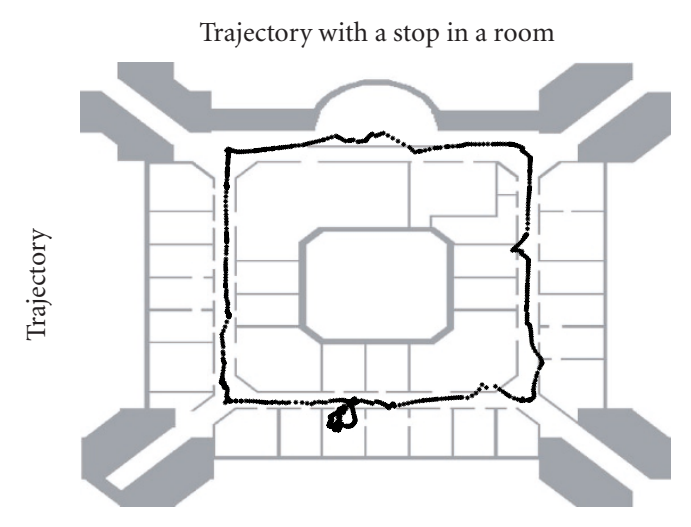

(b)

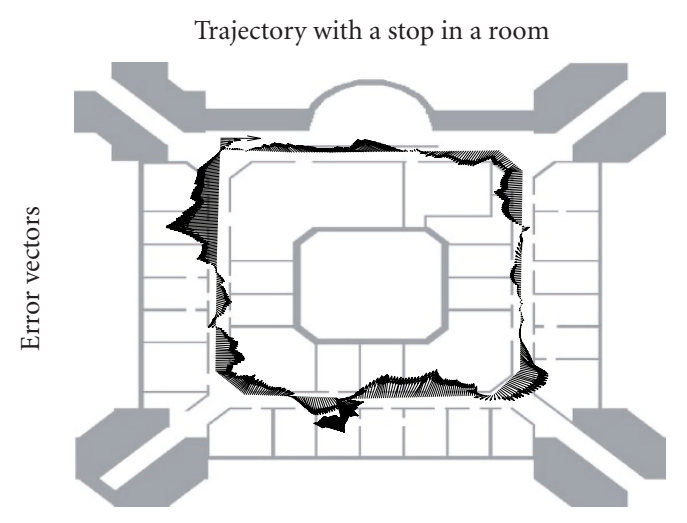

(d)

FIgURE 4: Trajectories obtained with a particle filter fed by some WiFi measurements. Pictures on the left present a trajectory along the corridor, and pictures on the right present the results for a trajectory in the corridor with a stop in a room.

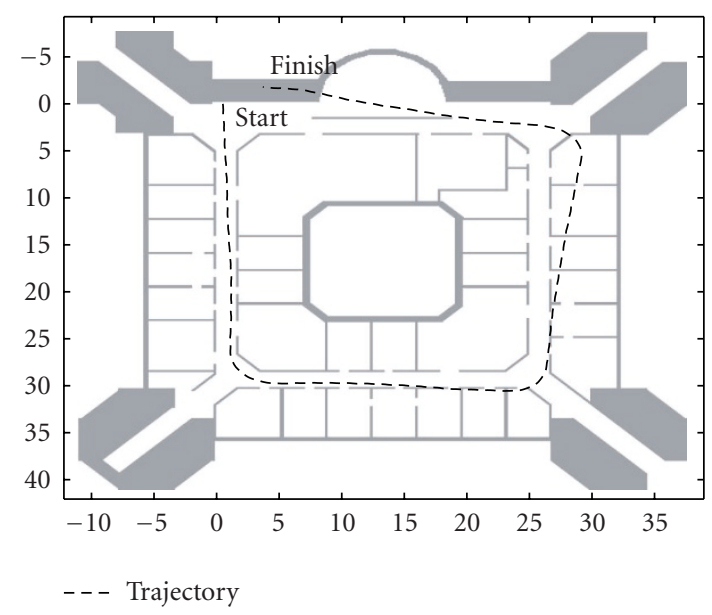

FIGURE 5: Trajectory obtained when just using dead-reckoning sensors.

contains the results of a trajectory in the corridor; whereas the right column contains the trajectory with a stop in a room. In this last simulation, the accelerometer and the gyroscope are both used to guide the particles through the building Figure 6 . It can be noticed that this combination of the WiFi positioning system with the data coming from the INS sensors seems to greatly improve the aspect of the final trajectory as merging those two techniques completely removes the wall crossings that were still a little bit visible when just positions from the WiFi positioning system were delivered.

Figure 7 proposes a performance comparison between all those positioning techniques. This figure presents the cumulative distributions of the instantaneous error that occurred after the filtering operations on the different data. These curves present the performances of the different systems, tried out to localize a mobile in our environment. It can be noticed that merging those two technologies enhanced the quality of the positioning results. This performance improvement mainly occurs when the filter has different choices especially at the end of a corridor. Delays appear in such a situation when just the WiFi positioning is used, but they are reduced when the particle filter has its particles guided with data coming from INS sensors. In fact, taking a decision in ambiguous situations is easier with the information coming from the INS sensors. Even though, all the indoor techniques prove to be relatively accurate depending on their complexity, but a 3-meter accuracy can be obtained for the 


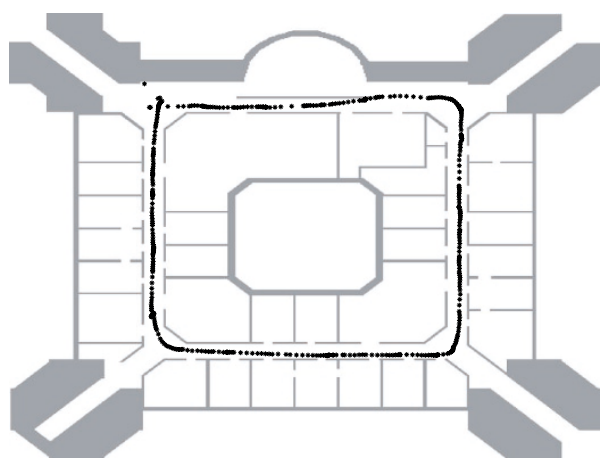

(a) Trajectory along the corridor.

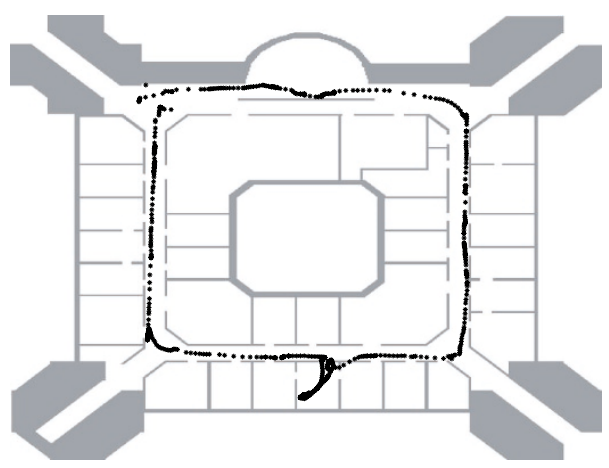

(b) Trajectory with a stop in a room.

FIGURE 6: Result of the fusion of the particle filter for the WiFi positioning and the inertial navigation system.

simplest ones, and a meter accuracy can be obtained for the most complex techniques (particle filter fusing information from a WiFi network and INS sensors). Those performances (Table 1) using such technologies seem very interesting as they can be applied and used in many applications, and the separation between accuracy and room correctness that existed in the first version of indoor WiFi positioning systems, starts disappearing when merging those relevant and simple information.

Tables 1 and 2 give a brief overview of the performances obtained with different indoor positioning systems. Filtering techniques implemented in our system allow a gain of $1.32 \mathrm{~m}$ for a Kalman filter and $2.02 \mathrm{~m}$ with a particle filter when just the WiFi measurements can be used for the positioning operation. Fusing INS information in the particle filter brings another improvement as the RMS error is then $1.53 \mathrm{~m}$ (compared to the $3.88 \mathrm{~m}$ presented in [25]). Fusing INS information in a WiFi system has several advantages. First, it improves the performances of the whole system in terms of positioning, and then it allows the device to be tracked when WiFi is unavailable (dead-reckoning navigation).

\section{CONCLUSIONS}

Indoor positioning based on WiFi infrastructure delivers interesting results with a low density of access points in the environments. Regarding to the performances that are awaited

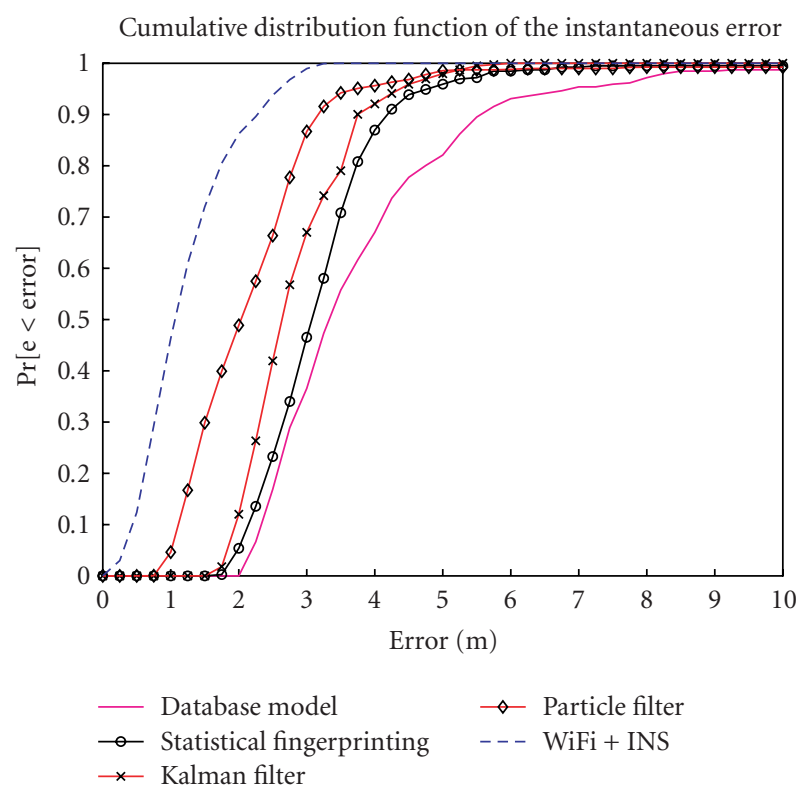

FIGURE 7: Trajectory obtained when just using dead-reckoning sensors.

TABLE 1: Comparison of the performances of the different systems for a trajectory in the corridor (use of 4 Access Points, located at each corner in the building, for the WiFi positioning).

\begin{tabular}{l|ccccc}
\hline & $\begin{array}{c}\text { Closest } \\
\text { neighbor }\end{array}$ & $\begin{array}{c}\text { Statistical } \\
\text { method }\end{array}$ & $\begin{array}{c}\text { Kalman } \\
\text { filter }\end{array}$ & $\begin{array}{c}\text { Particle } \\
\text { filter }\end{array}$ & $\begin{array}{c}\text { Particle } \\
\text { filter } \\
+ \text { INS }\end{array}$ \\
\hline $\begin{array}{l}\text { Mean } \\
\text { error }(\mathrm{m})\end{array}$ & 3.32 & 2.88 & 2.56 & 1.86 & 1.53 \\
\hline
\end{tabular}

TABLE 2: Positioning performances from other systems [25].

\begin{tabular}{l|cccc}
\hline & $\begin{array}{c}\text { Closest } \\
\text { neighbor } \\
\text { (RADAR) }\end{array}$ & $\begin{array}{c}\text { Propagation } \\
\text { model }\end{array}$ & $\begin{array}{c}\text { Propagation } \\
\text { model }+ \\
\text { RADAR }\end{array}$ & $\begin{array}{c}\text { Trilateration } \\
\text { (simple } \\
\text { model) }\end{array}$ \\
\hline $\begin{array}{l}\text { Mean } \\
\text { error }(\mathrm{m})\end{array}$ & 3.88 & 4.91 & 3.88 & 5.73 \\
\hline
\end{tabular}

from the technology, different techniques can be applied. For the most complex one, fusing information from the WiFi network, with information coming from inertial navigation sensors, it is possible to get performances close to the meter accuracy. This emerging technology is investing the current market, and such a positioning system should be available in the coming years on the mass market. However, the fingerprinting technique requires a received signal strength database which is time consuming to obtain for large building. Future work will consist in reducing the time process to build the database. Inertial navigation and the particle filter should be two key elements of the future system which will enable to build the database on the fly, assuming that an old database could be available or a very sparse database. 


\section{REFERENCES}

[1] “Breaking news: Canada mandates 911 for VoIP," telecomweb, April 2005, http://www.telecomweb.com/news/1112721769 .htm.

[2] P. Bahl and V. N. Padmanabhan, "RADAR: an in-building RFbased user location and tracking system," in Proceedings of 19th Annual Joint Conference of the IEEE Computer and Communications Societies (INFOCOM '00), vol. 2, pp. 775-784, Tel Aviv, Israel, March 2000.

[3] Y. Chen and H. Kobayashi, "Signal strength based indoor geolocation," in Proceedings of the IEEE International Conference on Communications (ICC '02), vol. 1, pp. 436-439, New York, NY, USA, April-May 2002.

[4] G. Welch and G. Bishop, "An introduction to the kalman filter," Tech. Rep., University of North Carolina, Chapel Hill, NC, USA, 2001.

[5] R. E. Kalman, "A new approach to linear filtering and prediction problems," Transactions of the ASME-Journal of Basic Engineering, vol. 82, pp. 35-45, 1960.

[6] M. S. Arulampalam, S. Maskell, N. Gordon, et al., "A tutorial on particle filters for online nonlinear/non-Gaussian Bayesian tracking," IEEE Transactions on Signal Processing, vol. 50, no. 2, pp. 174-188, 2002.

[7] F. Gustafsson, F. Gunnarsson, N. Bergman, et al., "Particle filters for positioning, navigation, and tracking," IEEE Transactions on Signal Processing, vol. 50, no. 2, pp. 425-437, 2002.

[8] A. Doucet, N. de Freitas, and N. Gordon, Sequential MonteCarlo Methods in Practice, Statistics for Engineering and Information Science, Springer, New York, NY, USA, 2001.

[9] P.-Y. Gilliéron, D. Buchel, I. Spassov, et al., "Indoor navigation performance analysis," in Proceedings of the 8th European Navigation Conference (GNSS '04), Rotterdam, The Netherlands, May 2004.

[10] P.-Y. Gilliéron and B. Merminod, "Personal navigation system for indoor applications," in Proceedings of the 11th IAIN World Congress, Berlin, Germany, October 2003.

[11] A. J. Motley and J. M. P. Keenan, "Personal communication radio coverage in buildings at $900 \mathrm{MHz}$ and $1700 \mathrm{MHz}$," Electronics Letters, vol. 24, no. 12, pp. 763-764, 1988.

[12] R. Vaughan and J. B. Andersen, Channels, Propagation and Antennas for Mobile Communications, Electromagnetic Waves Series 50, The Institution of Electrical Engineers, London, UK, 2003.

[13] A. Smailagic and D. Kogan, "Location sensing and privacy in a context-aware computing environment," IEEE Wireless Communications, vol. 9, no. 5, pp. 10-17, 2002.

[14] R. Battiti, T. L. Nhat, and A. Villani, "Location-aware computing: a neural network model for determining location in wireless LANs," Tech. Rep., Department of Information and Communication Technology, University of Trento, Trento, Italy, February 2002.

[15] A. Hatami and K. Pahlavan, "A comparative performance evaluation of RSS-based positioning algorithms used in WLAN networks," in Proceedings of the IEEE Wireless Communications and Networking Conference (WCNC '05), vol. 4, pp. 23312337, New Orleans, La, USA, March 2005.

[16] T. Roos, P. Myllymäki, H. Tirri, P. Misikangas, and J. Sievänen, "A probabilistic approach to WLAN user location estimation," International Journal of Wireless Information Networks, vol. 9, no. 3, pp. 155-164, 2002.

[17] T. Roos, P. Myllymäki, and H. Tirri, "A statistical modeling approach to location estimation," IEEE Transactions on Mobile Computing, vol. 1, no. 1, pp. 59-69, 2002.
[18] C. Musso, N. Oudjane, and F. L. Gland, "Improving regularized particle filters," in Sequential Monte Carlo Methods in Practice, chapter 12, Statistics for Engineering and Information Science, pp. 247-271, Springer, New York, NY, USA, 2001.

[19] F. Evennou, F. Marx, and E. Novakov, "Map-aided indoor mobile positioning system using particle filter," in Proceedings of the IEEE Wireless Communications and Networking Conference (WCNC '05), vol. 4, pp. 2490-2494, New Orleans, La, USA, March 2005.

[20] L. Liao, D. Fox, J. Hightower, et al., "Voronoi tracking: location estimation using sparse and noisy sensor data," in Proceeding of the IEEE/RSJ International Conference on Intelligent Robots and Systems (IROS '03), vol. 1, pp. 723-728, Las Vegas, Nev, USA, October 2003.

[21] Samsung, "Samsung introduces world's first "3-dimensional movement recognition" phone," Website, January 2005.

[22] J. V. Iribarne, Atmospheric Thermodynamics, chapter VII, D.Reidel, Dordrecht, Holland, 1973.

[23] A. Beiser, Earth Sciences, chapter 2, McGraw-Hill, New York, NY, USA, 1975.

[24] "Atmospheric pressure," http://www.scubageek.com/geek/articles/wwwatm.html.

[25] M. Robinson and I. Psaromiligkos, "Received signal strength based location estimation of a wireless LAN client," in Proceedings of the IEEE Wireless Communications and Networking Conference (WCNC '05), vol. 4, pp. 2350-2354, New Orleans, La, USA, March 2005.

Frédéric Evennou received a Diploma from INSA of Rennes (Institut National des Sciences Appliqués) in 2002. He is pursuing his Ph.D. degree at IMEP in Grenoble and at France Telecom R\&D. His recent research has been focused on indoor geolocation and tracking techniques. He is involved in European collaborative research projects, like the IST LIAISON project.

François Marx graduated from Ecole Polytechnique and from ENST in 1999 and 2001, respectively. Since 2001, he has been an R\&D Engineer with France Telecom R\&D and currently leads R\&D effort on software and cognitive radio as a Project Leader. His main activity is in digital signal processing for wireless systems and indoor geolocation. He is involved in a number of European and French collaborative research projects.
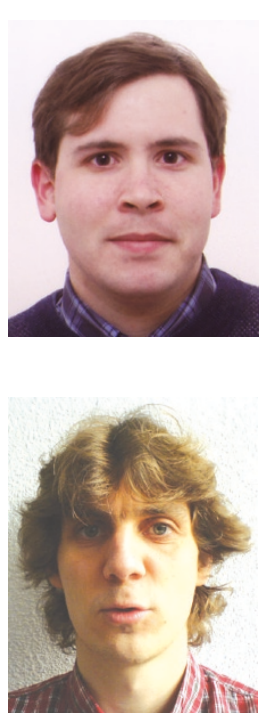